\title{
Structual Effects on Thermal Rearrangement of Fulleroids to Methanofullerenes. The Prominent Role of Cyclopropyl vs Aryl Substituent
}

Hiroshi Kitamura and Takumi Oshima*

Department of Applied Chemistry, Graduate School of Engineering, Osaka University, Suita, Osaka 565-0871, Japan

E-Mail: oshima@chem.eng.osaka-u.ac.jp

\section{Contents}

Synthesis of cyclopropyl trifluoromethyl ketone tosylhydrazone, and fulleroids $\mathbf{1 f}$ and $\mathbf{1 g}$ S2

Characterization of $\mathbf{2 c}(=\mathbf{d}), \mathbf{2 f}, \mathbf{2 g}$. S4

The ${ }^{1} \mathrm{H}-\mathrm{NMR}$ and ${ }^{13} \mathrm{C}$-NMR spectra of $\mathbf{1 f}, \mathbf{1 g}, \mathbf{2 c}(=\mathbf{d}), \mathbf{2 f}, \mathbf{2 g}$. S5

General procedure for kinetic study of the rearrangement of $\mathbf{1}$ and kinetic data. S17 
The cyclopropyl substituted fulleroids $\mathbf{1 b}$-e were synthesized and purified according to our previous report (ref. 1c). The fulleroids $\mathbf{1 h}$-j were prepared by the literature methods (Suzuki, T.; Li, Q.; Khemani, K. C.; Wudl, F.; Almarsson, O. Science 1991, 254, 1186; Shi, S.; Khemani, K. C.; Li, Q.; Wudl, F. J. Am. Chem. Soc. 1992, 114, 10656). Trifluoromethyl- and cyclopropyl-substituted fulleroid $\mathbf{1 f}$ and dicyclopropyl-subsituted fulleroid $\mathbf{1 g}$ were synthesized as follows.

\section{Cyclopropyl trifuloromethyl ketone tosylhydrazone}

$\alpha$-(Trifluoroacetyl)- $\gamma$-butyrolactone hydrate was synthesized according to the literature procedure by Archer et.al. ${ }^{1}$ To a solution of $\alpha$-(trifluoroacetyl)- $\gamma$-butyrolactone hydrate $(8.8 \mathrm{~g})$ in hexamethylphosphoamide (10ml) was added litium bromide $(12 \mathrm{~g})$, and heated at $160^{\circ} \mathrm{C}$ with distilling the generated ketone fraction for $1 \mathrm{~h}$. Further distillation of the obtained ketone gave the almost pure cyclopropyl trifluoromethyl ketone (2g, 33\%).

To a solution of cyclopropyl trifluoromethyl ketone $(0.5 \mathrm{~g})$ in EtOH $(2 \mathrm{~mL})$ was added tosylhydrazine $(0.67 \mathrm{~g}, 1.0$ eq) and refluxed for $1 \mathrm{~h}$. Concentration and recrystallization from EtOH provided pure cyclopropyl trifuloromethyl ketone tosylhydrazone $(0.4 \mathrm{~g}, 36 \%)$.

${ }^{1} \mathrm{H}-\mathrm{NMR}\left(\mathrm{CDCl}_{3}, 270 \mathrm{MHz}\right) \delta 8.39(1 \mathrm{H}), 7.84(\mathrm{~d}, \mathrm{~J}=7.4 \mathrm{~Hz}, 2 \mathrm{H}), 7.34(\mathrm{~d}, \mathrm{~J}=7.4 \mathrm{~Hz}, 2 \mathrm{H}), 2.44(\mathrm{~s}, 3 \mathrm{H}), 1.23(\mathrm{tt}, \mathrm{J}$ $=8.4,5.4 \mathrm{~Hz}, 1 \mathrm{H}), 1.01(\mathrm{~m}, 2 \mathrm{H}), 0.79(\mathrm{~m}, 2 \mathrm{H}){ }^{13} \mathrm{C}-\mathrm{NMR}\left(\mathrm{CDCl}_{3}, 67.5 \mathrm{MHz}\right) \delta 144.62(\operatorname{arom~C}), 142.47(1 \mathrm{C})$, 134.44(arom C), 129.58(arom 2CH), 127.79(arom $2 \mathrm{CH}), 119.87\left(\mathrm{CF}_{3}\right), 21.62\left(\mathrm{CH}_{3}\right), 5.46(\mathrm{CH}), 5.06\left(\mathrm{CH}_{2}\right)$

${ }^{1}$ S. Archer, C. Perianayagam, J. Med. Chem. 1979, 22, 306-309

\section{Cyclopropyl- and trifluoromethyl-substituted fulleroid 1f}

To a solution of $\mathrm{C}_{60}(20 \mathrm{mg}, 0.03 \mathrm{mmol})$ and the cyclopropyl trifluoromethyl ketone tosylhydrazone (1.0 eq.) in $o$-dichlorobenzene $(2 \mathrm{~mL})$ was added powdered $60 \% \mathrm{NaH}(1.1$ eq.) in mineral oil. The reaction solution was stirred for $24 \mathrm{~h}$ at $60^{\circ} \mathrm{C}$ in the dark. The reaction mixture was loaded onto silicagel column chromatography and eluted with toluene to remove the inorganic materials. Monoadducts were separated from $\mathrm{C}_{60}$ and the multiadducts by HPLC on Buckyprep column with toluene. The total yield of monoadducts was $19 \%$ (4.5mg, fulleroid 1f: methanofullerenes $\mathbf{2 f}=3: 4$ ). The fulleroid $\mathbf{1 f}$ and methanofullerene $2 \mathbf{f}$ were separated by the short-time recycle HPLC treatment $(\sim 1 \mathrm{~h})$ on Buckyprep column with toluene.

Compound 1f LC-MS (APPI) m/z 842, ${ }^{1} \mathrm{H}-\mathrm{NMR}\left(1: 1 \mathrm{CS}_{2}: \mathrm{CDCl}_{3}, 270 \mathrm{MHz}\right) \delta 2.90(\mathrm{tt}, \mathrm{J}=8.4,5.9 \mathrm{~Hz}, 1 \mathrm{H})$, $1.50-1.30(\mathrm{~m}, 4 \mathrm{H}){ }^{13} \mathrm{C}-\mathrm{NMR}\left(1: 1 \mathrm{CS}_{2}: \mathrm{CDCl}_{3}, 100 \mathrm{MHz}\right) \delta 146.96(2 \mathrm{C}), 145.18(2 \mathrm{C}), 144.60(1 \mathrm{C}), 144.56(2 \mathrm{C})$, 144.07(2C), 143.86(2C), 143.82(2C), 143.80(4C), 143.65(2C), 143.56(2C), 143.04(3C), 142.96(4C), 142.83(2C), 142.75(2C), 142.22(2C), 142.14(2C), 142.08(2C), 141.79(2C), 141.15(2C), 141.02(3C), 140.19(2C), 138.36(2C), 137.80(2C), 137.77(2C), 136.08(2C), 135.88(1C), 134.91(2C), 128.71(2C), 52.78(1C), 24.68(CH), 10.72(2 $\left.\mathrm{CH}_{2}\right)$ ${ }^{19} \mathrm{~F}-\mathrm{NMR}\left(1: 1 \mathrm{CS}_{2}: \mathrm{CDCl}_{3}, 376.48 \mathrm{MHz}, \mathrm{CF}_{3} \mathrm{COOH}\right.$ (internal standard)) $\delta-13.65 \mathrm{UV} / \mathrm{vis}$ (o-dichlorobenzene) $\lambda_{\max } 538,603 \mathrm{~nm}$ 


\section{Dicyclopropyl-substituted fulleroid $1 \mathrm{~g}$}

To a solution of $\mathrm{C}_{60}(50 \mathrm{mg}, 0.07 \mathrm{mmol})$ and the dicyclopropyl ketone hydrazone (1.5 eq.) in $o$-dichlorobenzene (5mL) was added powdered $\mathrm{Ag}_{2} \mathrm{O}$ (1.0 eq.). The reaction solution was stirred for $2 \mathrm{~h}$ at room temperature in the dark. The reaction mixture was loaded onto Florisil column chromatography and eluted with toluene to remove the inorganic materials. Then, the monoadducts of fulleroid $\mathbf{1 g}$ and methanofullerene $\mathbf{2 g}$ (trace) were clearly separated from $\mathrm{C}_{60}$ and the multiadducts by HPLC on Buckyprep column with toluene. The yield of the monoadducts was $35 \%$ (20mg).

Compound 1g LC-MS (APPI) m/z 814, ${ }^{1} \mathrm{H}-\mathrm{NMR}\left(1: 1 \mathrm{CS}_{2}: \mathrm{CDCl}_{3}, 270 \mathrm{MHz}\right) \delta 2.29(\mathrm{tt}, \mathrm{J}=8.6,5.9 \mathrm{~Hz}, 1 \mathrm{H})$, $1.40-1.23(\mathrm{~m}, 4 \mathrm{H}), 0.87-0.74(\mathrm{~m}, 3 \mathrm{H}), 0.61(\mathrm{~m}, 2 \mathrm{H}){ }^{13} \mathrm{C}-\mathrm{NMR}\left(1: 1 \mathrm{CS}_{2}: \mathrm{CDCl}_{3}, 67.5 \mathrm{MHz}\right) \delta 144.93(2 \mathrm{C})$, 144.43(2C), 143.73(2C), 143.51(3C), 143.44(3C), 143.37(2C), 143.17(2C), 142.79(2C), 142.76(4C), 142.57(2C), 142.48(2C), 142.29(1C), 142.24(4C), 142.03(2C), 141.69(4C), 141.64(2C), 141.49(2C), 141.29(4C), 141.04(2C), 140.42(2C), 140.10(2C), 139.92(1C), 138.30(2C), 138.10 (2C), 137.66(2C), 137.51(2C), 135.14(1C), 134.47(2C), 52.78(1C), 23.16(CH), 20.47(CH), 9.51( $\left(\mathrm{CH}_{2}\right), 6.78\left(\mathrm{CH}_{2}\right) \mathrm{UV} / \mathrm{vis}$ (o-dichlorobenzene) $\lambda_{\max } 546,603 \mathrm{~nm}$ 
Characterization of $\mathbf{2 c}(=\mathbf{d}), \mathbf{f}, \mathbf{g}$

2c (= 2d) LC-MS (APPI) m/z 788, ${ }^{1} \mathrm{H}-\mathrm{NMR}\left(1: 1 \mathrm{CS}_{2}: \mathrm{CDCl}_{3}, 270 \mathrm{MHz}\right) \delta 2.29$ (tt, J = 8.6, $\left.5.6 \mathrm{~Hz}, 1 \mathrm{H}\right), 1.97$ (s, $3 \mathrm{H}), 1.12(\mathrm{~m}, 2 \mathrm{H}), 0.97(\mathrm{~m}, 2 \mathrm{H}){ }^{13} \mathrm{C}-\mathrm{NMR}\left(1: 1 \mathrm{CS}_{2}: \mathrm{CDCl}_{3}, 100 \mathrm{MHz}\right) \delta 148.54(2 \mathrm{C}), 148.43(2 \mathrm{C}), 145.49(4 \mathrm{C})$, 144.97(4C), 144.84(3C), 144.83(2C), 144.71(1C), 144.52(2C), 144.50(2C), 144.12(1C), 143.95(3C), 143.54(2C), 143.48(2C), 142.93(3C), 142.89(2C), 142.87(2C), 142.82(2C), 142.79(2C), 142.00(2C), 141.98(2C), 141.95(4C), 140.77(2C), 140.71(2C), 137.44(2C), 137.24(2C), $82.97(2 \mathrm{C}), 40.21(1 \mathrm{C}), 13.36\left(\mathrm{CH}_{3}\right), 12.77(\mathrm{CH}), 3.94\left(2 \mathrm{CH}_{2}\right)$ UV/vis (o-dichlorobenzene) $\lambda_{\max } 435,496,697 \mathrm{~nm}$

Compound 2f LC-MS (APPI) m/z 842, ${ }^{1} \mathrm{H}-\mathrm{NMR}\left(1: 1 \mathrm{CS}_{2}: \mathrm{CDCl}_{3}, 270 \mathrm{MHz}\right) \delta 2.15(\mathrm{tt}, \mathrm{J}=8.6,5.8 \mathrm{~Hz}, 1 \mathrm{H})$, $1.39-1.30(\mathrm{~m}, 2 \mathrm{H}), 1.16(\mathrm{~m}, 2 \mathrm{H}){ }^{13} \mathrm{C}-\mathrm{NMR}\left(1: 1 \mathrm{CS}_{2}: \mathrm{CDCl}_{3}, 100 \mathrm{MHz}\right) \delta 146.32(2 \mathrm{C}), 145.17(2 \mathrm{C}), 145.14(5 \mathrm{C})$, 145.12(2C), 145.04(2C), 144.80(2C), 144.68(2C), 144.63(2C), 144.57(6C), 144.52(1C), 144.32(2C), 143.78(2C), 143.67(2C), 143.12(2C), 143.07(1C), 143.03(1C), 142.99(2C), 142.94(2C), 142.83(2C), 142.16(2C), 142.11(2C), 141.47(2C), 141.41(2C), 140.79(4C), 140.22(2C), 138.22(2C), 74.10(2C), 46.71(1C), 12.36(CH), 8.59(2 $\left.\mathrm{CH}_{2}\right)$ ${ }^{19} \mathrm{~F}-\mathrm{NMR}\left(1: 1 \mathrm{CS}_{2}: \mathrm{CDCl}_{3}, 376.48 \mathrm{MHz}, \mathrm{CF}_{3} \mathrm{COOH}\right.$ (internal standard)) $\delta-16.09 \quad \mathrm{UV} / \mathrm{vis}$ (o-dichlorobenzene) $\lambda_{\max } 429,492,690 \mathrm{~nm}$

Compound 2g LC-MS (APPI) m/z 814, ${ }^{1} \mathrm{H}-\mathrm{NMR}\left(1: 1 \mathrm{CS}_{2}: \mathrm{CDCl}_{3}, 270 \mathrm{MHz}\right) \delta 1.98(\mathrm{tt}, \mathrm{J}=8.6,5.6 \mathrm{~Hz}, 2 \mathrm{H}), 1.30$ $(\mathrm{m}, 4 \mathrm{H}), 1.03(\mathrm{~m}, 4 \mathrm{H}){ }^{13} \mathrm{C}-\mathrm{NMR}\left(1: 1 \mathrm{CS}_{2}: \mathrm{CDCl}_{3}, 67.5 \mathrm{MHz}\right) \delta 148.24(4 \mathrm{C}), 145.10(4 \mathrm{C}), 144.73(4 \mathrm{C}), 144.65(4 \mathrm{C})$, 144.57(2C), 144.29(4C), 144.01(2C), 143.77(4C), 143.39(4C), 142.82(2C), 142.61(8C), 141.81(4C), 141.64(4C), 140.48(4C), 137.54(4C), 82.26(2C), 47.03(1C), 15.67(CH), 7.67(2 $\left.\mathrm{CH}_{2}\right) \mathrm{UV} / \mathrm{vis}$ (o-dichlorobenzene) $\lambda_{\max } 435$, $500,701 \mathrm{~nm}$ 
Q

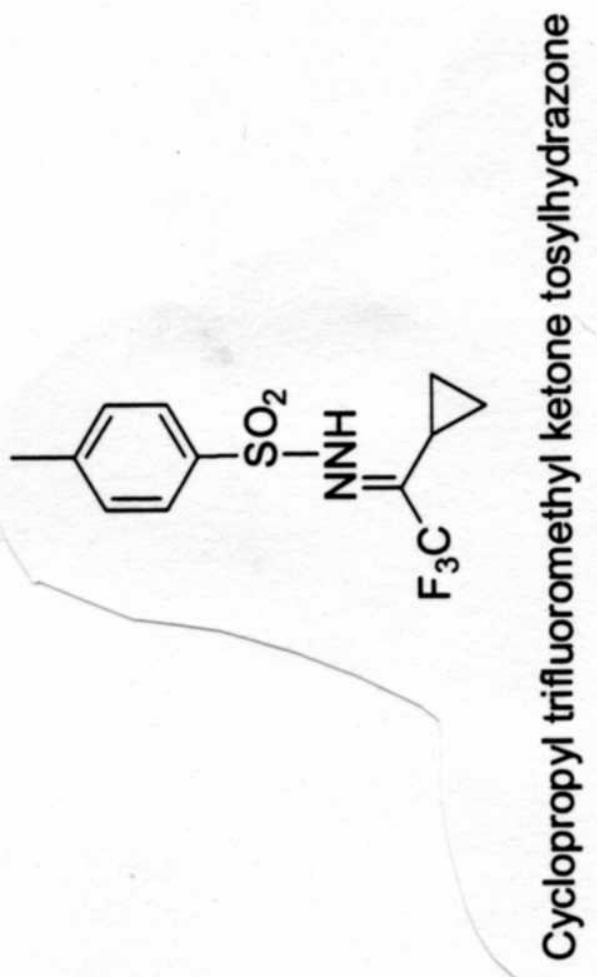

$\frac{3}{\frac{3}{5}}$ 
D. 




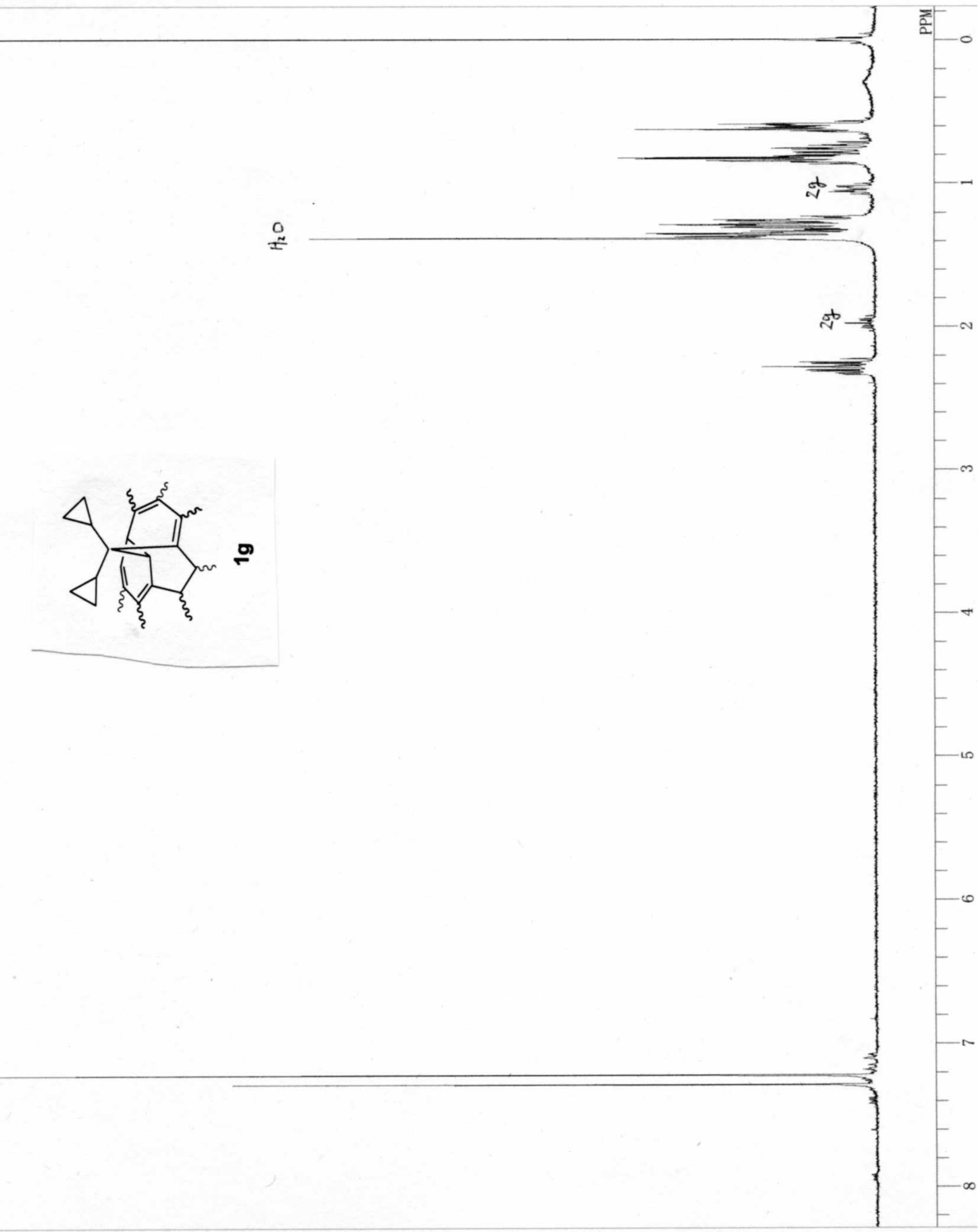




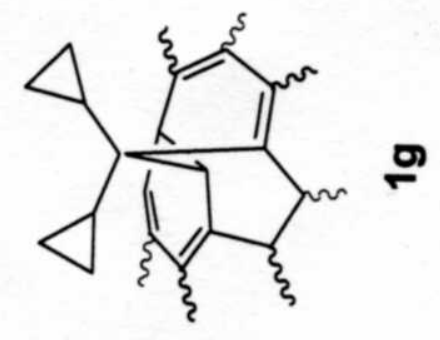


온

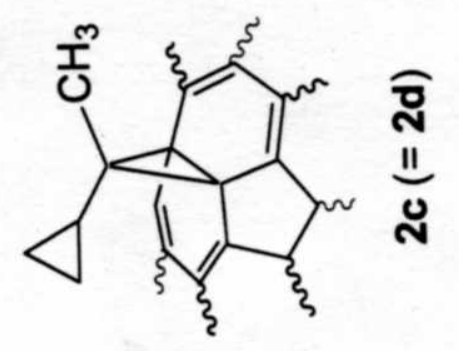




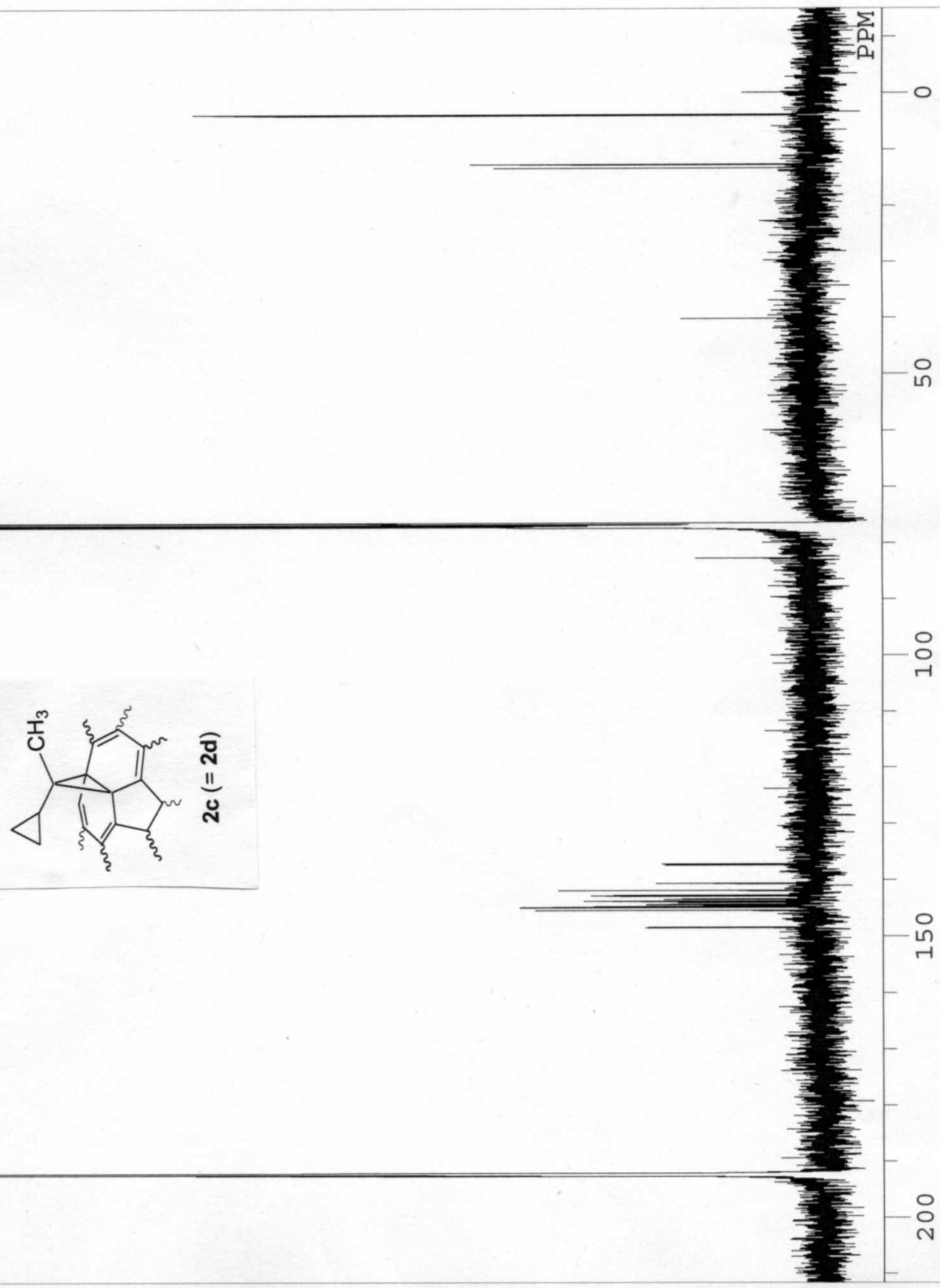




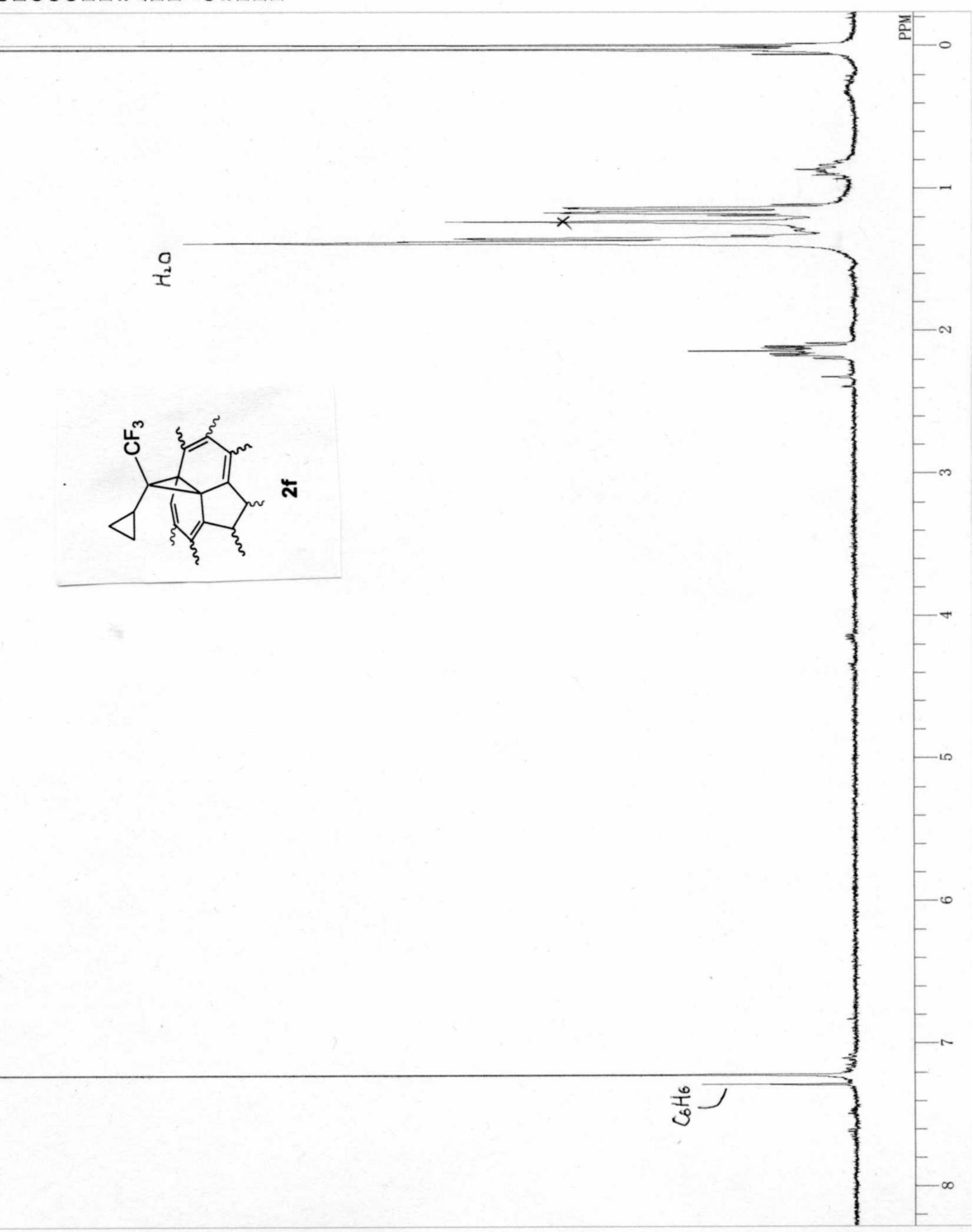





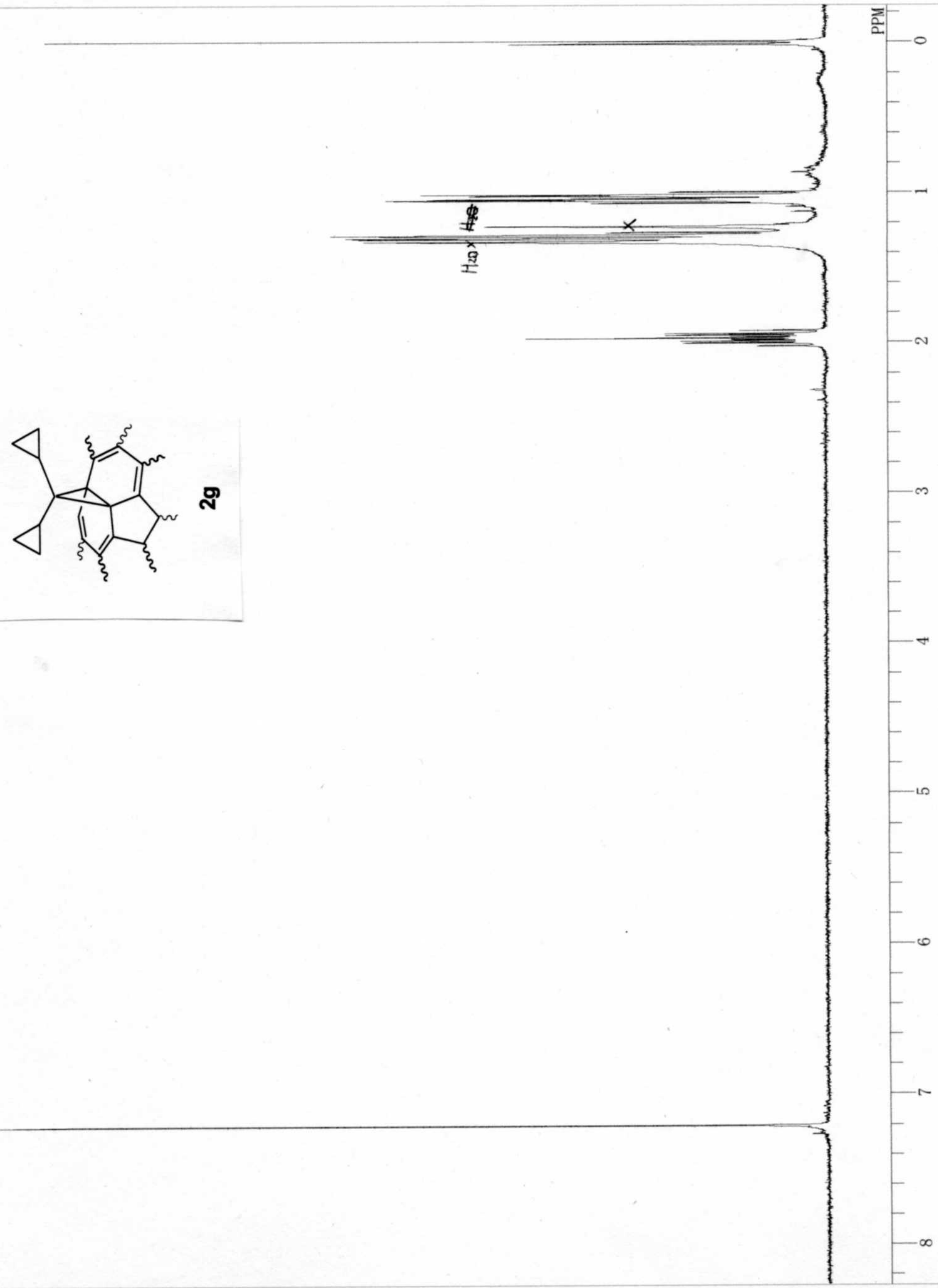




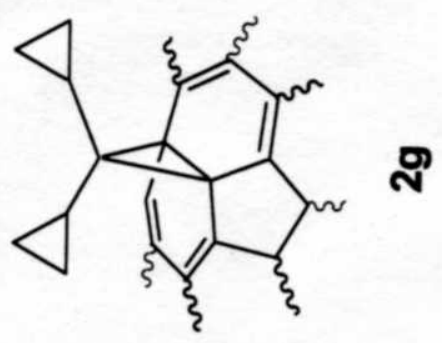




\section{General procedure for kinetic study of the thermal rearrangement of 1}

For 1b-e, a 5-mm NMR tube, wrapped with aluminium foil, was charged with the [6,5] open isomer (contaminated with $[6,5]$ open diastereoisomer and a trace amount of $[6,6]$ closed isomer ) and 0.75 $\mathrm{mL}$ of ODCB- $d 4$ (ca. 2-10 mM) and was sealed. For 1f-j, a 5-mL screw-capped test tube, wrapped with aluminium foil, was charged with the [6,5] open isomer (contaminated with a trace amount of [6,5] open diastereoisomer and [6,6] closed isomer) and $2 \mathrm{~mL}$ of ODCB (ca. 0.15-0.30 mM). For $\mathbf{1 h}$, $2 \mathrm{~mL}$ of ODCB solution was added $0.2 \mathrm{mM}$ of pyrene as an internal standard. Kinetic studies of the rearrangement of the $[6,5]$ open to the [6,6] closed isomer were performed in ODCB- $d_{4}$ by ${ }^{1} \mathrm{H}-\mathrm{NMR}$ or in usual ODCB by HPLC on a Buckeyprep column by monitoring the decrease of $[6,5]$ isomer.

The progress of thermal rearrangement was carried out by heating the sample solution with a thermostatted bath $\left( \pm 0.05^{\circ} \mathrm{C}\right)$. Since these rearrangements provided only the $[6,6]$ closed isomer as the rearranged product, the first-order rate constants $(k)$ were obtained by plotting logarithmic values of the relative signal intensities of the diagnostic proton(s) for ${ }^{1} \mathrm{H}$ NMR and of the relative absorption peak intensity $(310 \mathrm{~nm})$ of a constant aliquot $(10 \mathrm{~mL})$ of a reaction solution for HPLC.

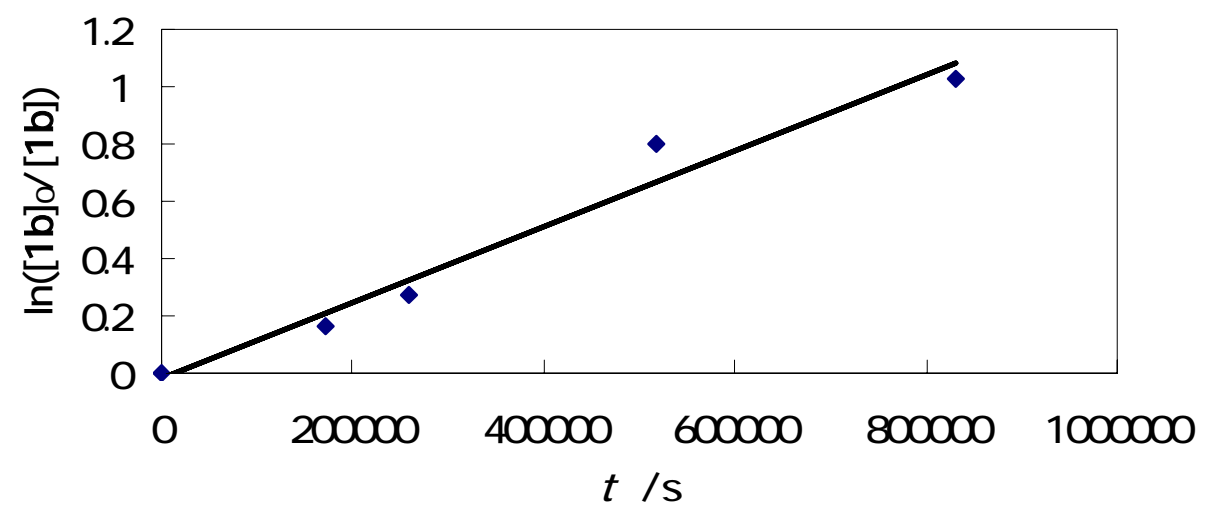

Figure $S$-1. Plots of $\ln \left([\mathbf{1 b}]_{0} /[\mathbf{1 b}]\right)$ vs $t$

\begin{tabular}{c|cc} 
& $\ln \left([\mathbf{1 b}]_{0} /[\mathbf{1 b}]\right)=1.328 \mathrm{E}-06 t-1.988 \mathrm{E}-02$ & $\left(R^{2}=9.681 \mathrm{E}-01\right)$ \\
$t / \mathrm{s}$ & $\ln \left([\mathbf{1 b}]_{0} /[\mathbf{1 b}]\right)$ \\
\hline 0 & 0 \\
172800 & 0.168032 \\
259200 & 0.272644 \\
518400 & 0.797595 \\
831600 & 1.028724
\end{tabular}




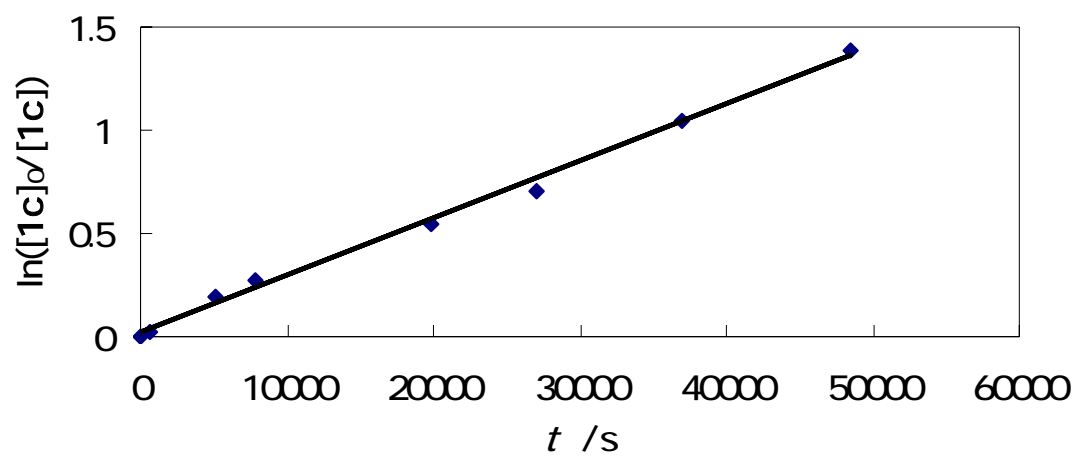

Figure $S$-2. Plots of $\ln \left([\mathbf{1 c}]_{0} /[1 \mathrm{c}]\right)$ vs $t$

\begin{tabular}{c|c|c|c}
\multicolumn{4}{c}{$\ln \left([\mathbf{1 c}]_{0} /[\mathbf{1 c}]\right)=2.769 \mathrm{E}-05 t+1.895$} \\
$t / \mathrm{s}$ & $\ln \left([\mathbf{1 c}]_{0} /[\mathbf{1 c}]\right)$ & $t / \mathrm{s}$ & $\ln \left([\mathbf{1 c}]_{0} /[\mathbf{1 c}]\right)$ \\
\hline 0 & 0 & 27000 & 0.70641 \\
660 & 0.024845 & 36900 & 1.050504 \\
5040 & 0.197014 & 48480 & 1.386598 \\
7860 & 0.277936 & & \\
19800 & 0.544436 & &
\end{tabular}

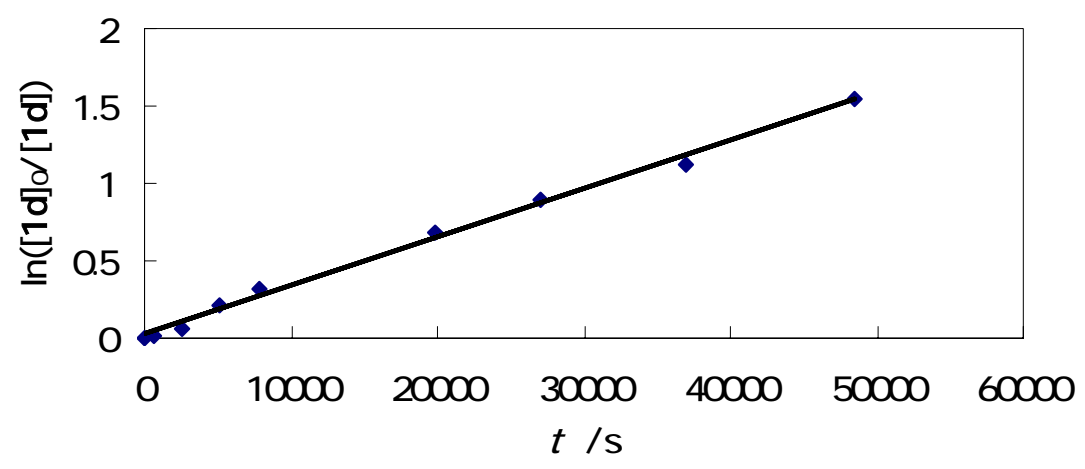

Figure $S$-3. Plots of $\ln \left([\mathbf{1 d}]_{0} /[\mathbf{1 d}]\right)$ vs $t$

$$
\ln \left([\mathbf{1 d}]_{0} /[\mathbf{1 d}]\right)=3.121 \mathrm{E}-05 t+2.520 \mathrm{E}-02\left(R^{2}=9.949 \mathrm{E}-01\right)
$$

\begin{tabular}{c|c|c|c}
$t / \mathrm{s}$ & $\ln \left([\mathbf{1 d}]_{0} /[\mathbf{1 d}]\right)$ & $t / \mathrm{s}$ & $\ln \left([\mathbf{1 d}]_{0} /[\mathbf{1 d}]\right)$ \\
\hline 0 & 0 & 19800 & 0.685515 \\
660 & 0.020885 & 27000 & 0.886512 \\
2520 & 0.057761 & 36900 & 1.123127 \\
5040 & 0.212567 & 48480 & 1.542603 \\
7860 & 0.325726 & &
\end{tabular}




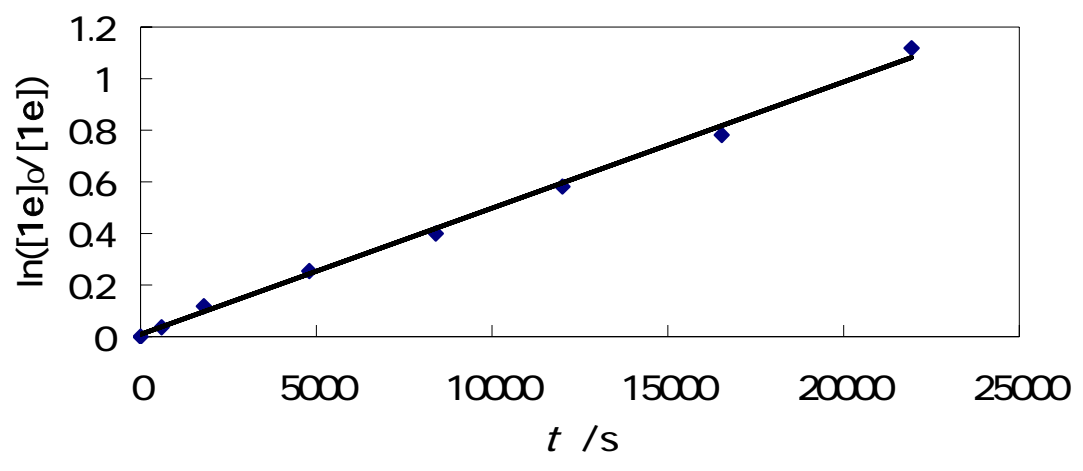

Figure $S$-4. Plots of $\ln \left([1 \mathrm{e}]_{0} /[1 \mathrm{e}]\right)$ vs $t$

\begin{tabular}{c|c|c|c}
\multicolumn{4}{|c}{$\ln \left([\mathbf{1 e}]_{0} /[\mathbf{1 e}]\right)=4.908 \mathrm{E}-05 t+6.510$} \\
$t / \mathrm{s}$ & $\ln \left([\mathbf{1 e}]_{0} /[\mathbf{1 e}]\right)$ & $t / \mathrm{s}$ & $\ln \left([\mathbf{1 e}]_{0} /[\mathbf{1 e}]\right)$ \\
\hline 0 & 0 & 16500 & 0.783292 \\
600 & 0.037095 & 21900 & 1.11569 \\
1800 & 0.120709 & \\
4800 & 0.251911 & \\
8400 & 0.397369 & \\
12000 & 0.584975
\end{tabular}

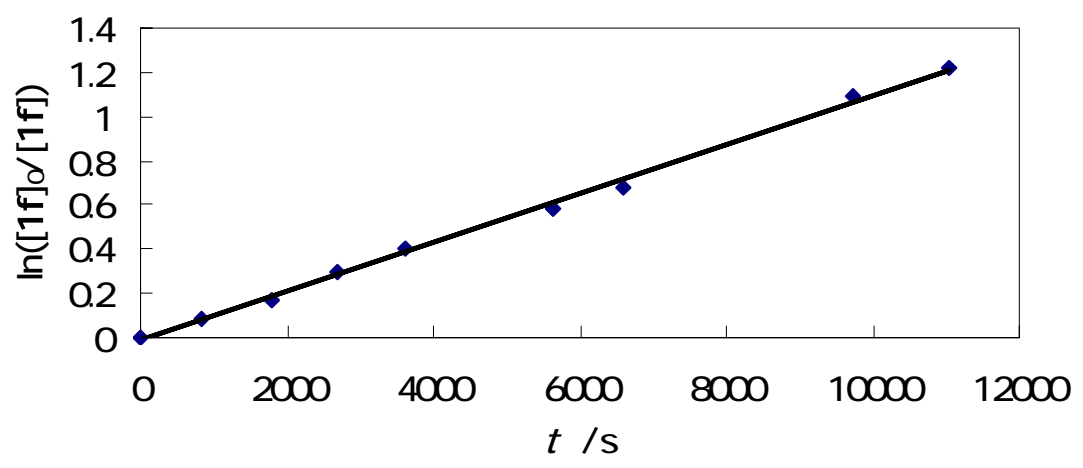

Figure $S$-5. Plots of $\ln \left([\mathbf{1 f}]_{0} /[\mathbf{1 f}]\right)$ vs $t$

$\ln \left([\mathbf{1 f}]_{0} /[\mathbf{1 f}]\right)=1.106 \mathrm{E}-04 t-1.382 \mathrm{E}-02(R 2=9.975 \mathrm{E}-01)$

\begin{tabular}{c|c|c|c}
$t / \mathrm{s}$ & $\ln \left([\mathbf{1 f}]_{0} /[\mathbf{1 f}]\right)$ & $t / \mathrm{s}$ & $\ln \left([\mathbf{1 f}]_{0} /[\mathbf{1 f}]\right)$ \\
\hline 0 & 0 & 5640 & 0.585464 \\
840 & 0.081908 & 6600 & 0.67545 \\
1800 & 0.173091 & 9720 & 1.087192 \\
2700 & 0.293128 & 11040 & 1.214475 \\
3600 & 0.402953 & &
\end{tabular}




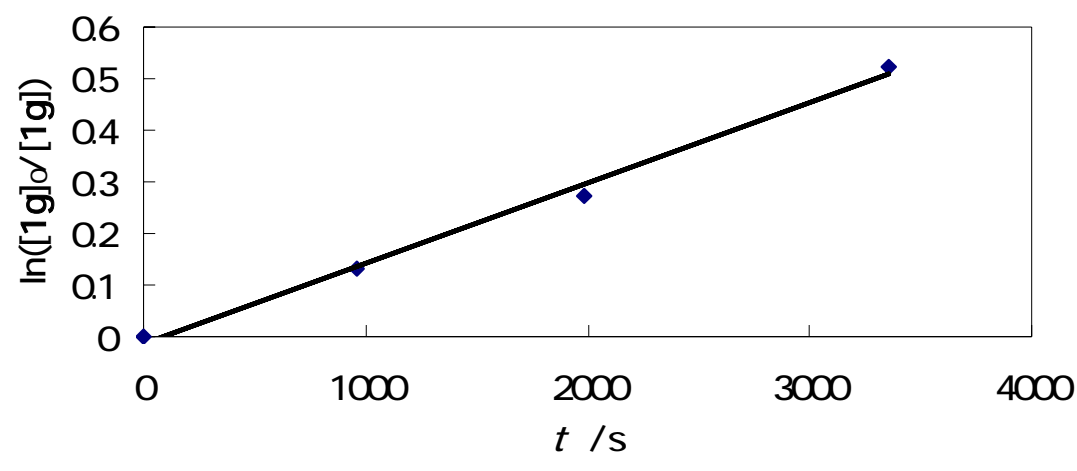

Figure S-6. Plots of $\ln \left([\mathbf{1 g}]_{0} /[\mathbf{1 g}]\right)$ vs $t$

$\ln \left([\mathbf{1 g}]_{0} /[\mathbf{1 g}]_{)}=1.551 \mathrm{E}-04 t-1.143 \mathrm{E}-02\left(R^{2}=9.947 \mathrm{E}-01\right)\right.$

\begin{tabular}{c|c}
$\begin{array}{c}110 \mathrm{oC} \\
t / \mathrm{s}\end{array}$ & $\ln \left([\mathbf{1 g}]_{0} /[\mathbf{1 g}]\right)$ \\
\hline 0 & 0 \\
960 & 0.133851 \\
1980 & 0.274036 \\
3360 & 0.523365
\end{tabular}

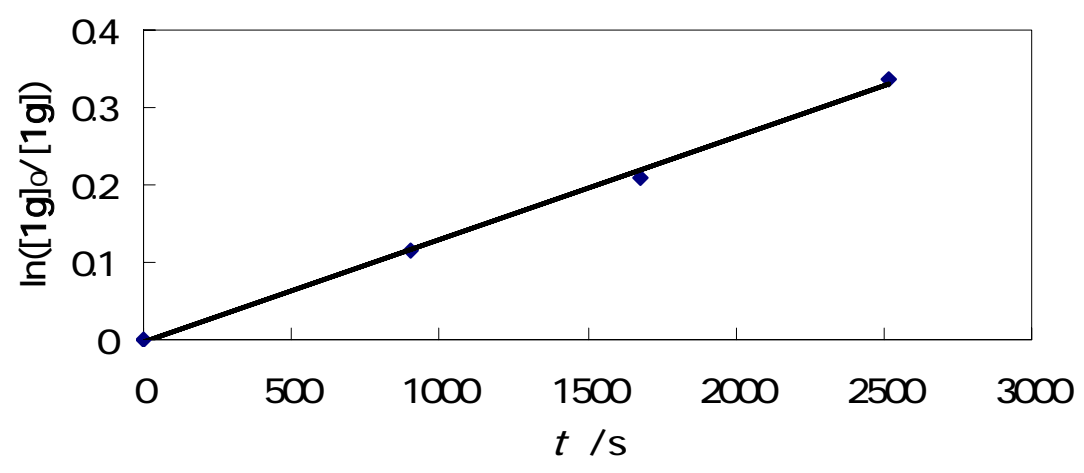

Figure $S$-7. Plots of $\ln \left([\mathbf{1 g}]_{0} /[\mathbf{1 g}]\right)$ vs $t$

$\ln \left([\mathbf{1 g}]_{0} /[\mathbf{1 g}]\right)=1.321 \mathrm{E}-04 t-3.729 \mathrm{E}-03(R 2=9.976 \mathrm{E}-01)$

\begin{tabular}{c|c}
$110 \mathrm{oC}$ & \\
$t / \mathrm{s}$ & $\ln \left([\mathbf{1 g}]_{0} /[\mathbf{1 g}]\right)$ \\
\hline 0 & 0 \\
900 & 0.114227 \\
1680 & 0.208752 \\
2520 & 0.335724
\end{tabular}




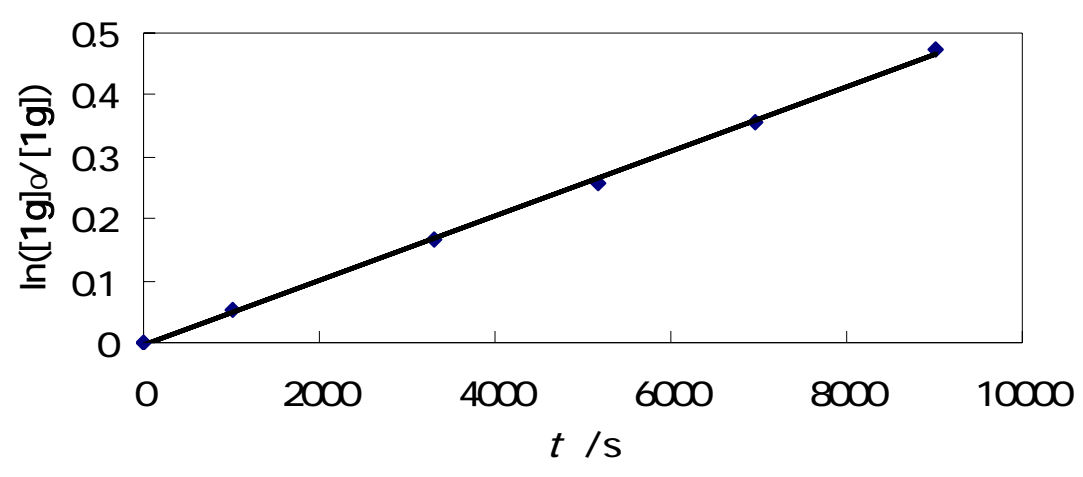

Figure $S$-8. Plots of $\ln \left([\mathbf{1 g}]_{0} /[\mathbf{1 g}]\right)$ vs $t$

$$
\ln \left([\mathbf{1 g}]_{0} /[\mathbf{1 g}]\right)=5.223 \mathrm{E}-05 t-3.608 \mathrm{E}-03\left(R^{2}=9.987 \mathrm{E}-01\right)
$$

\begin{tabular}{c|c}
$100 \mathrm{oC}$ & \\
$t / \mathrm{s}$ & $\ln \left([\mathbf{1 g}]_{0} /[\mathbf{1 g}]\right)$ \\
\hline 0 & 0 \\
1020 & 0.053088 \\
3300 & 0.165815 \\
5160 & 0.256528 \\
6960 & 0.356379 \\
9000 & 0.475211
\end{tabular}

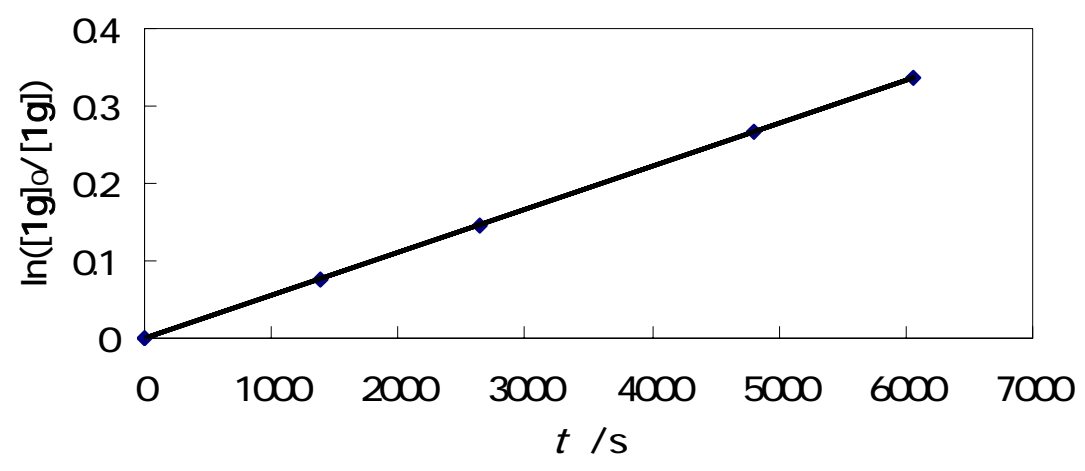

Figure S-9. Plots of $\ln \left([\mathbf{1 g}]_{0} /[\mathbf{1 g}]\right)$ vs $t$

$$
\ln \left([\mathbf{1 g}]_{0} /[\mathbf{1 g}]\right)=5.588 \mathrm{E}-05 t-9.457 \mathrm{E}-04(R 2=1.000 \mathrm{E}+00)
$$

$100 \mathrm{oC}$

\begin{tabular}{c|c|c|c}
$t / \mathrm{s}$ & $\ln \left([\mathbf{1 g}]_{0} /[\mathbf{1 g}]\right)$ & $t / \mathrm{s}$ & $\ln \left([\mathbf{1 g}]_{0} /[\mathbf{1 g}]\right)$ \\
\hline 0 & 0 & 6060 & 0.337517 \\
1380 & 0.075314 & & \\
2640 & 0.145725 & & \\
4800 & 0.268147 & &
\end{tabular}




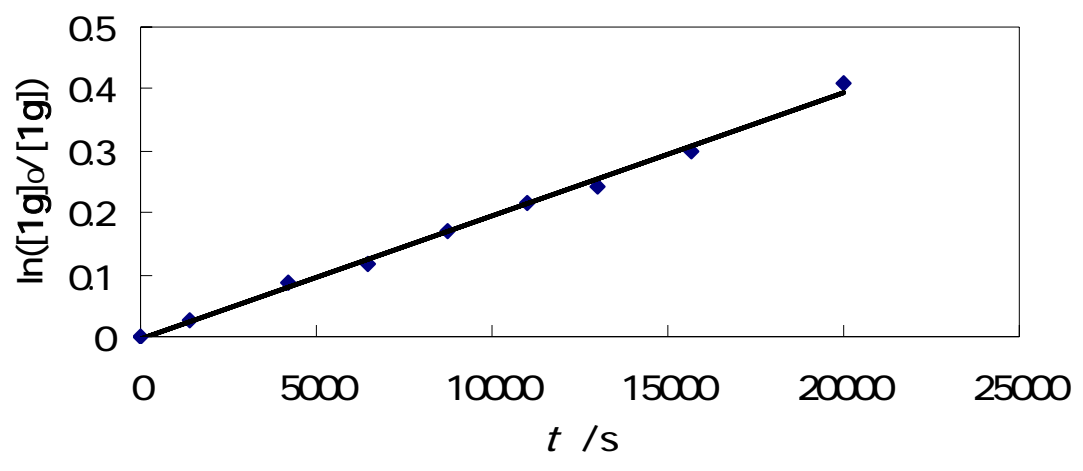

Figure $S$-10. Plots of $\ln \left([\mathbf{1 g}]_{0} /[\mathbf{1 g}]\right)$ vs $t$

$$
\ln \left([\mathbf{1 g}]_{0} /[\mathbf{1 g}]\right)=1.989 \mathrm{E}-05 t-3.030 \mathrm{E}-03\left(R^{2}=9.964 \mathrm{E}-01\right)
$$

\begin{tabular}{c|c|c|c}
$90 \mathrm{oC}$ & \multicolumn{3}{|c}{} \\
$t / \mathrm{s}$ & $\ln \left([\mathbf{1 g}]_{0} /[\mathbf{1 g}]\right)$ & $t / \mathrm{s}$ & $\ln \left([\mathbf{1 g}]_{0} /[\mathbf{1 g}]\right)$ \\
\hline 0 & 0 & 10980 & 0.217297 \\
1380 & 0.027189 & 13020 & 0.244226 \\
4200 & 0.08652 & 15660 & 0.300737 \\
6480 & 0.117797 & 19980 & 0.408117 \\
8760 & 0.171063 & &
\end{tabular}

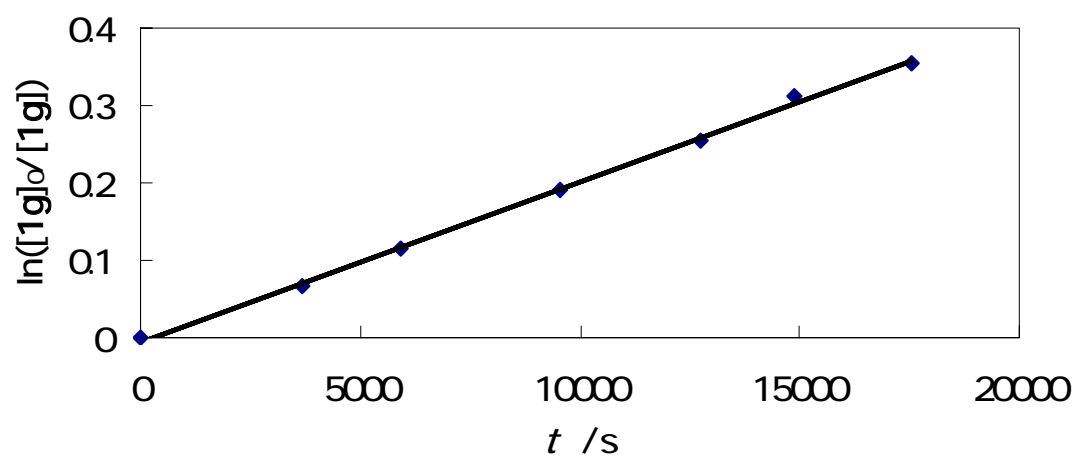

Figure S-11. Plots of $\ln \left([\mathbf{1 g}]_{0} /[\mathbf{1 g}]\right)$ vs $t$

$$
\ln \left([\mathbf{1 g}]_{0} /[\mathbf{1 g}]\right)=2.075 \mathrm{E}-05 t-5.550 \mathrm{E}-03\left(R^{2}=9.985 \mathrm{E}-01\right)
$$

\begin{tabular}{|c|c|c|c|}
\hline \multicolumn{4}{|l|}{$90 \mathrm{oC}$} \\
\hline$t / \mathrm{s}$ & $\ln \left([\mathbf{1 g}]_{0} /[\mathbf{1 g}]\right)$ & $t / \mathrm{s}$ & $\ln \left([\mathbf{1 g}]_{0} /[\mathbf{1 g}]\right)$ \\
\hline 0 & 0 & 12720 & 0.255291 \\
\hline 3660 & 0.06564 & 14880 & 0.312078 \\
\hline 5940 & 0.114673 & 17520 & 0.35517 \\
\hline 9540 & 0.191968 & & \\
\hline
\end{tabular}




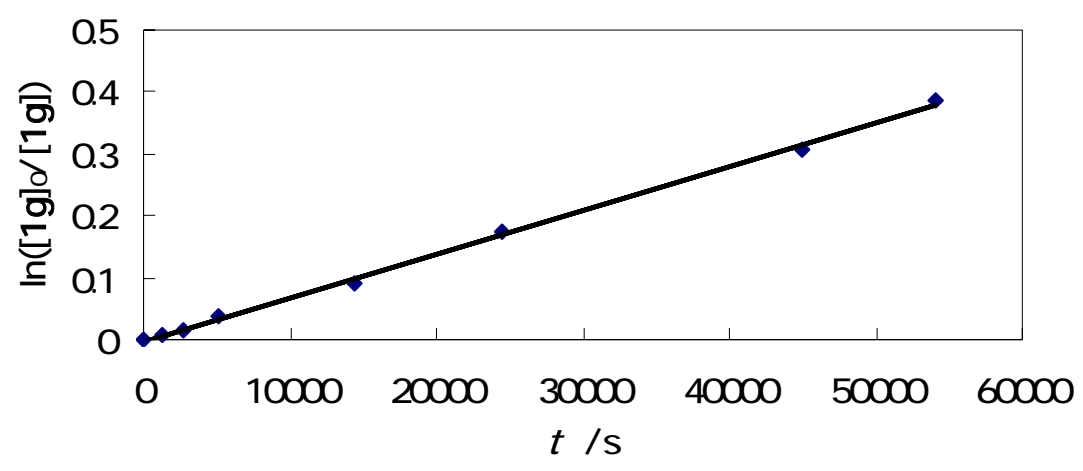

Figure $S$-12. Plots of $\ln \left([\mathbf{1 g}]_{0} /[\mathbf{1 g}]\right)$ vs $t$

$$
\ln \left([\mathbf{1 g}]_{0} /[\mathbf{1 g}]\right)=7.064 \mathrm{E}-06 t-2.246 \mathrm{E}-03\left(R^{2}=9.986 \mathrm{E}-01\right)
$$

$80 \mathrm{oC}$

\begin{tabular}{c|c|c|c}
$t / \mathrm{s}$ & $\ln \left([\mathbf{1 g}]_{\mathbf{0}} /[\mathbf{1 g}]\right)$ & $t / \mathrm{s}$ & $\ln \left([\mathbf{1 g}]_{0} /[\mathbf{1 g}]\right)$ \\
\hline 0 & 0 & 44880 & 0.307516 \\
1200 & 0.007887 & 54060 & 0.385889 \\
2640 & 0.016395 & & \\
5160 & 0.036035 & & \\
14460 & 0.090668 & & \\
24420 & 0.174794 &
\end{tabular}

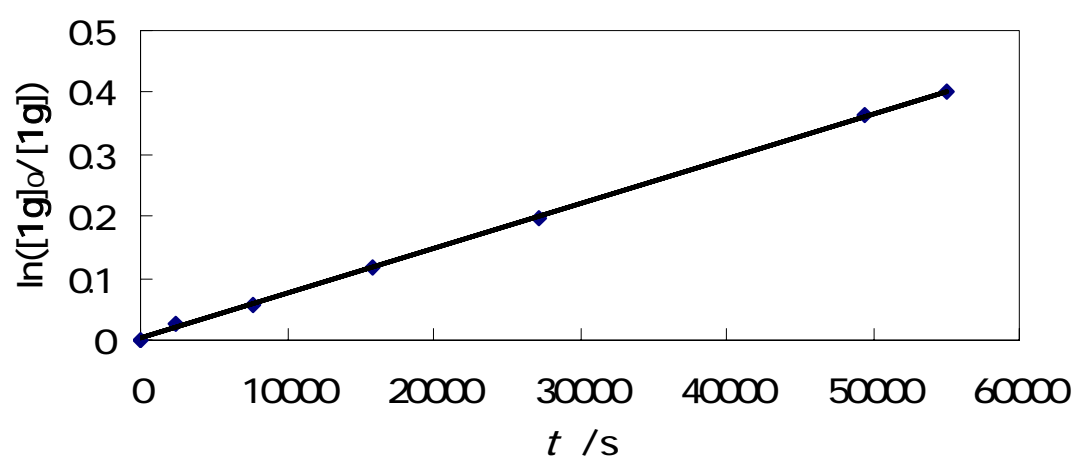

Figure $S$-13. Plots of $\ln \left([\mathbf{1 g}]_{0} /[\mathbf{1 g}]\right)$ vs $t$

$$
\ln \left([\mathbf{1 g}]_{0} /[\mathbf{1 g}]\right)=7.277 \mathrm{E}-06 t+2.018 \mathrm{E}-03(R 2=9.996 \mathrm{E}-01)
$$

$80 \mathrm{oC}$

\begin{tabular}{c|c|c|c}
$t / \mathrm{s}$ & $\ln \left([\mathbf{1 g}]_{0} /[\mathbf{1 g}]\right)$ & $t / \mathrm{s}$ & $\ln \left([\mathbf{1 g}]_{0} /[\mathbf{1 g}]\right)$ \\
\hline 0 & 0 & 27240 & 0.196217 \\
2400 & 0.024768 & 49380 & 0.363862 \\
7620 & 0.056186 & 55080 & 0.402554 \\
15840 & 0.117049 & &
\end{tabular}




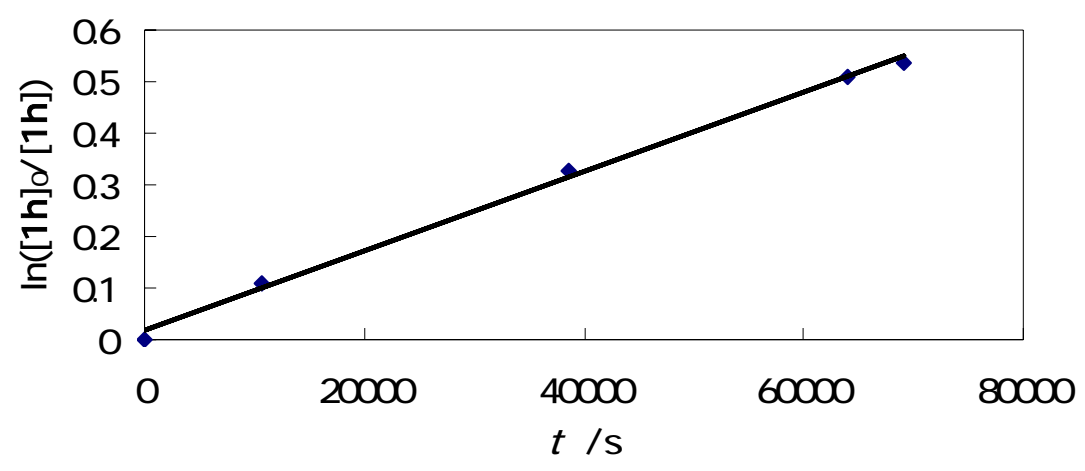

Figure S-14. Plots of $\ln \left([1 \mathrm{~h}]_{0} /[1 \mathrm{~h}]\right)$ vs $t$

\begin{tabular}{c|c} 
& $\ln \left([\mathbf{1 h}]_{0} /[\mathbf{1 h}]\right)$ \\
$t / \mathrm{s}$ & $\ln \left([\mathbf{1 h}]_{0} /[\mathbf{1 h}]\right)$ \\
\hline 0 & 0 \\
10560 & 0.111358 \\
38520 & 0.327988 \\
63900 & 0.510566 \\
69060 & 0.537302
\end{tabular}

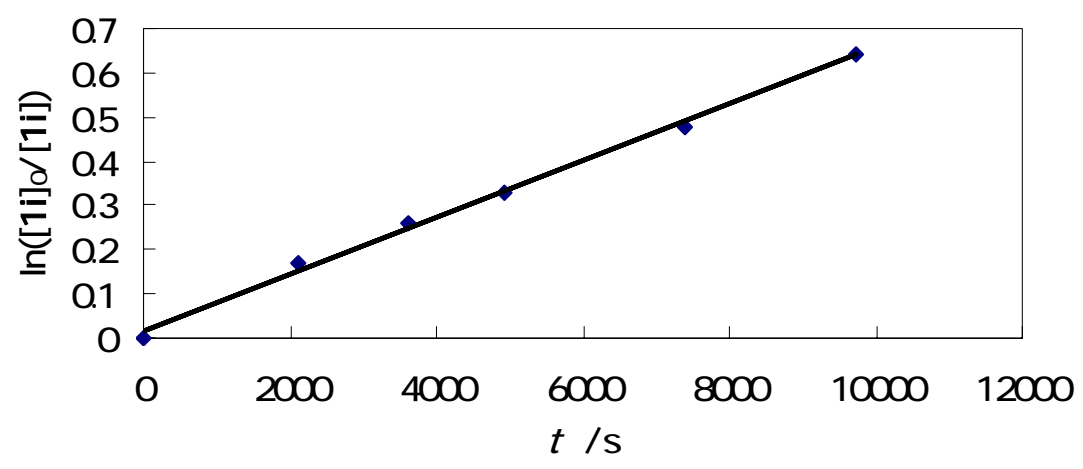

Figure $S$-15. Plots of $\ln \left([1 \mathbf{i}]_{0} /[1 \mathbf{i}]\right)$ vs $t$

$\ln \left([\mathbf{1 i}]_{0}[\mathbf{1 i}]\right)=6.423 \mathrm{E}-05 t+1.611 \mathrm{E}-02\left(R^{2}=9.969 \mathrm{E}-01\right)$

\begin{tabular}{c|c}
$t / \mathrm{s}$ & $\ln \left([\mathbf{1 i}]_{\mathbf{0}} /[\mathbf{1 i}]\right)$ \\
\hline 0 & 0 \\
2100 & 0.167739 \\
3600 & 0.258427 \\
4920 & 0.329236 \\
7380 & 0.479336 \\
9720 & 0.64229
\end{tabular}




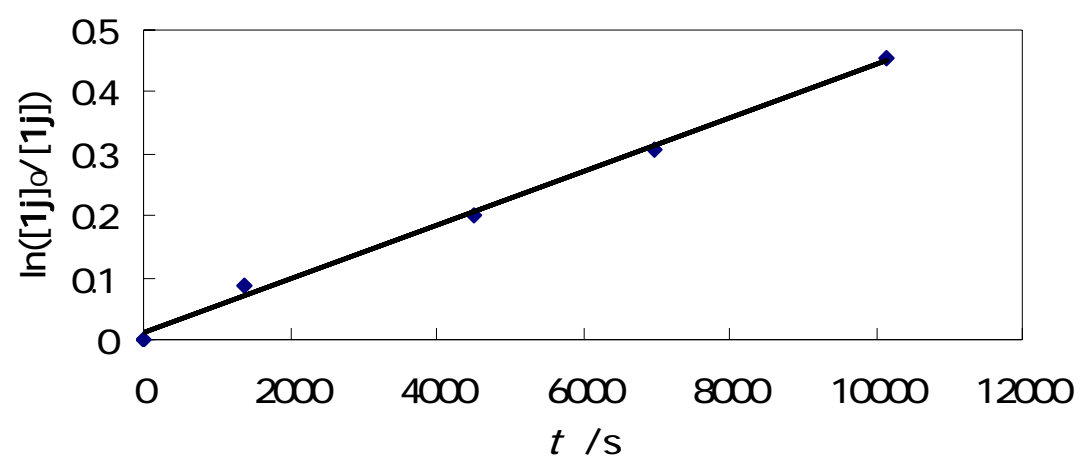

Figure $S$-16. Plots of $\ln \left([\mathbf{1} \mathbf{j}]_{0} /[\mathbf{1 j}]\right)$ vs $t$

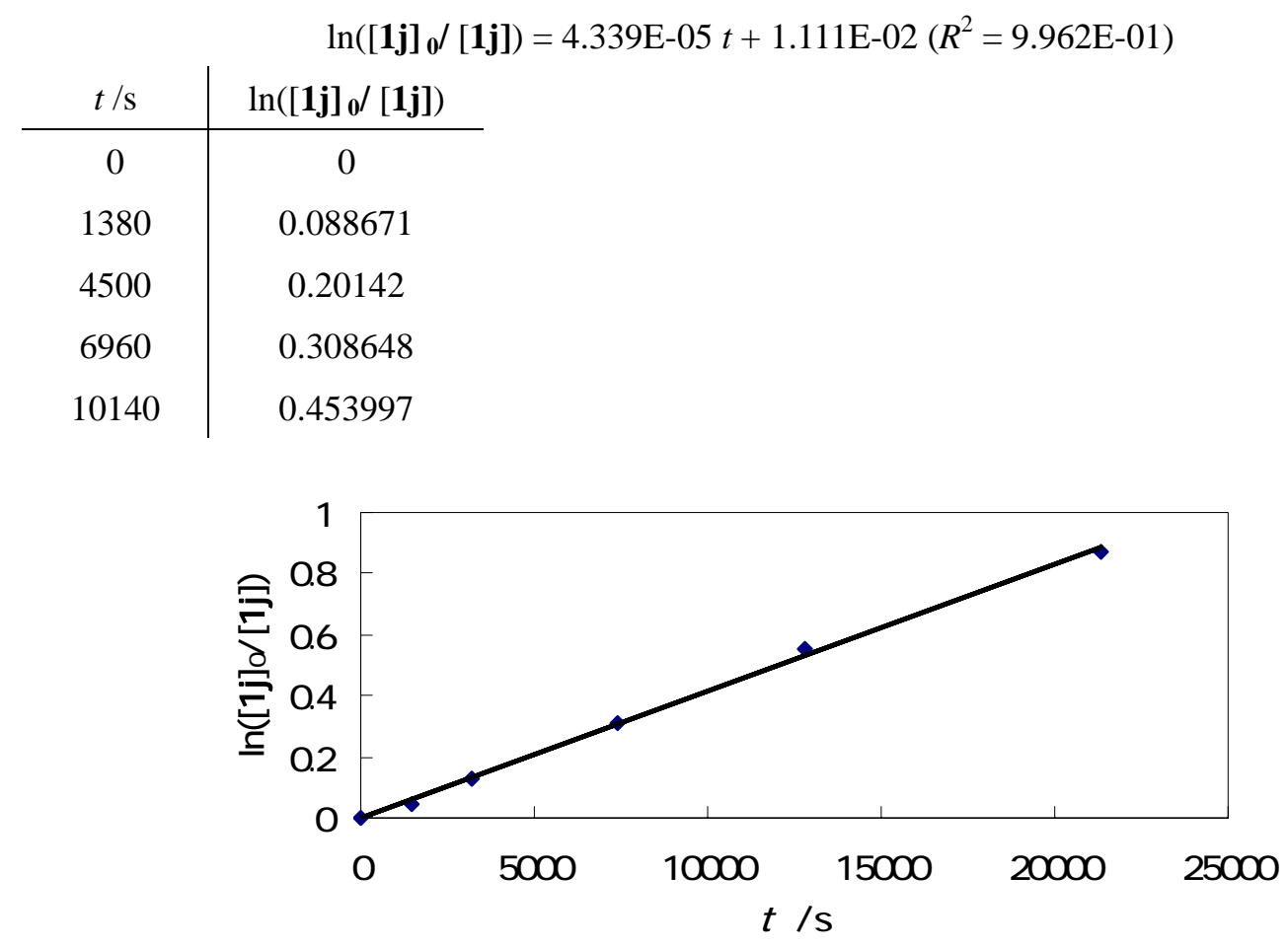

Figure $S$-17. Plots of $\ln \left([\mathbf{1} \mathbf{j}]_{0} /[\mathbf{1} \mathbf{j}]\right)$ vs $t$

\begin{tabular}{c|c} 
& $\ln \left([\mathbf{1} \mathbf{j}]_{\mathbf{0}} /[\mathbf{1} \mathbf{j}]\right)=4.156 \mathrm{E}-05 t-9.719 \mathrm{E}-04\left(R^{2}=9.982 \mathrm{E}-01\right)$ \\
$t / \mathrm{s}$ & $\ln \left([\mathbf{1} \mathbf{j}]_{\mathbf{0}} /[\mathbf{1} \mathbf{j}]\right)$ \\
\hline 0 & 0 \\
1440 & 0.046339 \\
3180 & 0.128834 \\
7380 & 0.310199 \\
12780 & 0.554726 \\
21360 & 0.871849
\end{tabular}




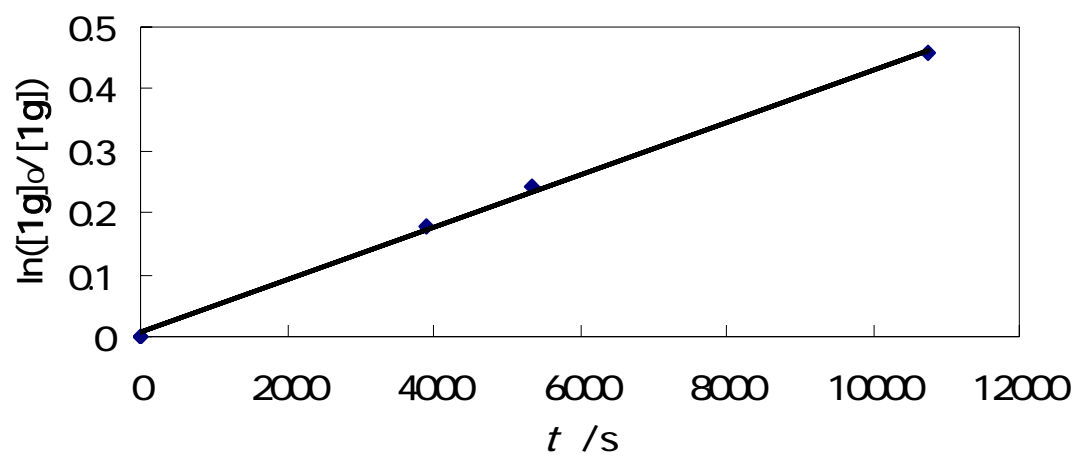

Figure $S$-18. Plots of $\ln \left([\mathbf{1 g}]_{0} /[\mathbf{1 g}]\right)$ vs $t$

$$
\ln \left([\mathbf{1 g}]_{0} /[\mathbf{1 g}]\right)=4.251 \mathrm{E}-05 t+6.410 \mathrm{E}-03\left(R^{2}=9.987 \mathrm{E}-01\right)
$$

$90 \mathrm{oC}$ in 1,1,2,2-tetrachloroethane

\begin{tabular}{c|c}
$t / \mathrm{s}$ & $\ln \left([\mathbf{1 g}]_{0} /[\mathbf{1 g}]\right)$ \\
\hline 0 & 0 \\
3900 & 0.176189 \\
5340 & 0.241145 \\
10740 & 0.457727
\end{tabular}

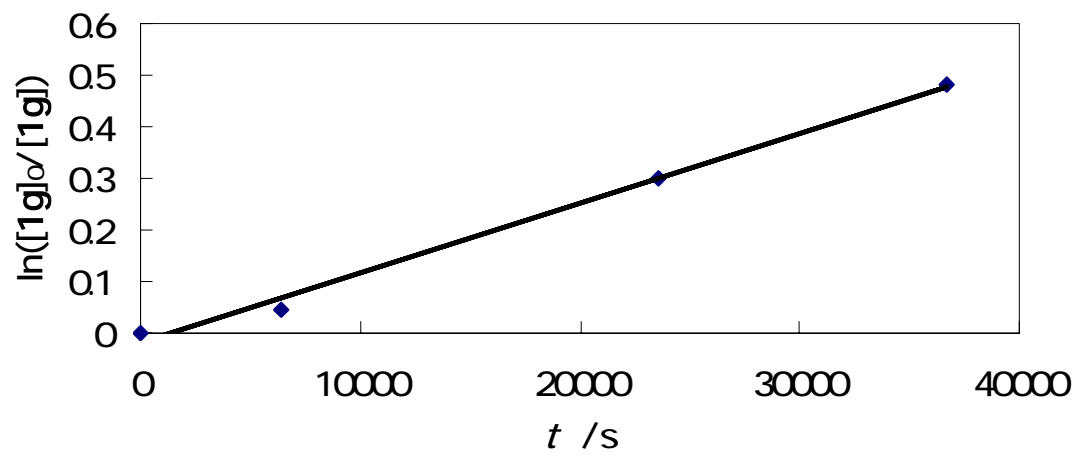

Figure $S$-19. Plots of $\ln \left([\mathbf{1 g}]_{0} /[\mathbf{1 g}]\right)$ vs $t$

$$
\ln \left([\mathbf{1 g}]_{0} /[\mathbf{1 g}]\right)=1.350 \mathrm{E}-05 t-1.940 \mathrm{E}-02\left(R^{2}=9.939 \mathrm{E}-01\right)
$$

$90 \mathrm{oC}$ in toluene

\begin{tabular}{c|c}
$t / \mathrm{s}$ & $\ln \left([\mathbf{1 g}]_{0} /[\mathbf{1 g}]\right)$ \\
\hline 0 & 0 \\
6420 & 0.044064 \\
23580 & 0.298405 \\
36720 & 0.480957
\end{tabular}




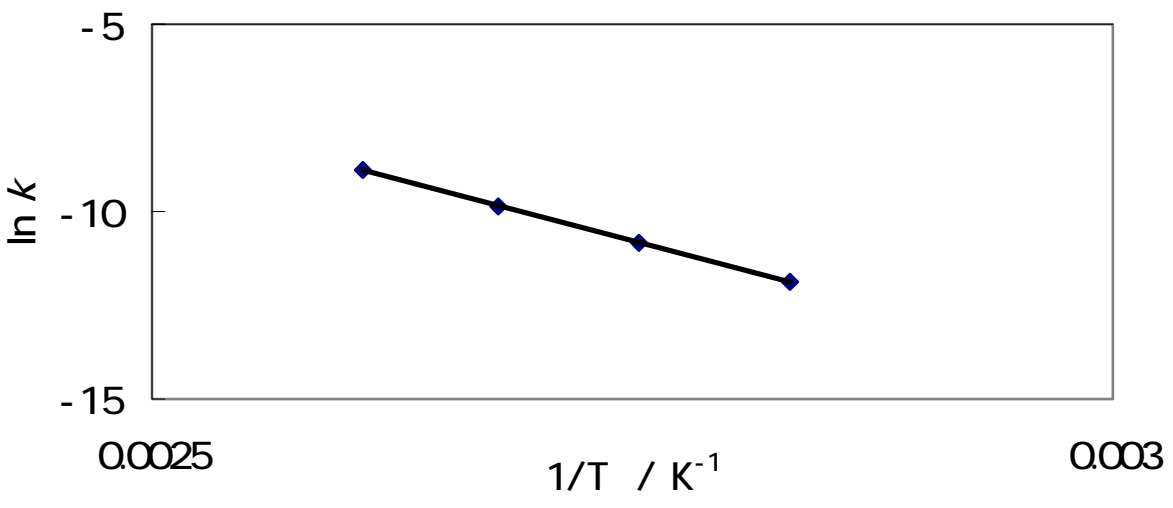

Figure S-20. Plots of $\ln k$ vs $1 / \mathrm{T}$

$$
\ln k=-1.344 \mathrm{E}+04 \times(1 / \mathrm{T})+2.622 \mathrm{E}+01\left(R^{2}=9.999 \mathrm{E}-01\right)
$$

$$
\begin{aligned}
\mathrm{Ea} & =1.344 \mathrm{E}+04 / \mathrm{J} \mathrm{mol}^{-1} \\
\ln \mathrm{A} & =2.622 \mathrm{E}+01 \mathrm{~J} \mathrm{~mol}^{-1} \mathrm{~K}^{-1}
\end{aligned}
$$

\begin{tabular}{c|cccc}
$\mathrm{T}\left({ }^{\circ} \mathrm{C}\right)$ & 80 & 90 & 100 & 110 \\
\hline$k$ & $7.17 \mathrm{E}-06$ & $2.03 \mathrm{E}-05$ & $5.40 \mathrm{E}-05$ & $1.42 \mathrm{E}-04$ \\
$\ln k$ & -11.8456 & -10.8049 & -9.82653 & -8.8596835 \\
$1 / \mathrm{T}$ & 0.002832 & 0.002754 & 0.00268 & 0.002609944
\end{tabular}

